\title{
CÂNFORA: UM BOM MODELO PARA ILUSTRAR TÉCNICAS DE RMN
}

Julliane Diniz Yoneda e Kátia Zaccur Leal*

Departamento de Físico-Química, Instituto de Química, Universidade Federal Fluminense, Outeiro de São João Batista, s/n, 24020-150 Niterói - RJ, Brasil

\section{Peter Rudolf Seidl}

Departamento de Processos Orgânicos, Escola de Química, Universidade Federal do Rio de Janeiro, 21949-900, Ilha do Fundão, Rio de Janeirp - RJ, Brasil

Rodrigo Bagueira de V. Azeredo

Departamento de Química Orgânica, Instituto de Química, Universidade Federal Fluminense, 24020-150 Niterói - RJ, Brasil Erich Kleinpeter

Universität Potsdam, Chemisches Institut, P.O. Box 6015 53, D-14415, Potsdam, Germany

Recebido em 30/11/06; aceito em 16/3/07; publicado na web em 9/11/07

\begin{abstract}
CAMPHOR: A GOOD MODEL FOR ILLUSTRATING NMR TECHNIQUES. The use of Nuclear Magnetic Resonance spectroscopy to establish the three-dimensional structures of molecules is an important component of modern Chemistry courses. The combination of techniques that can be used for this purpose is conveniently illustrated by their application to the camphor molecule. This paper presents applications of several techniques used in NMR spectral interpretation in an increasing order of complexity. The result of individual experiments is illustrated in order to familiarize the user with the way connectivity through bonds and through space is established from 1D/2D-NMR spectra and molecular stereochemistry is determined from different NMR experiments.
\end{abstract}

Keywords: organic chemistry; NMR spectroscopy; camphor.

\section{INTRODUÇÃO}

A cânfora (Figura 1) é uma cetona terpenóide obtida da árvore cânfora, encontrada no Oriente e na América do Sul, e representa uma classe de compostos bioativos que são sintetizados por plantas. É um bom exemplo de molécula utilizada no cotidiano que pode servir para induzir estudantes de química a exercitar seu raciocínio na identificação de monoterpenos desconhecidos por Ressonância Magnética Nuclear (RMN) ${ }^{2}$. A cânfora tem um cheiro forte e penetrante, gosto amargo e é ligeiramente fria ao tato. Ela tem se revelado como excelente excitante em casos de parada cardíaca causada por doenças ou resultantes de febres infecciosas, tais como tifóide e pneumonia. A cânfora é utilizada internamente por sua influência calmante em histeria, nervosismo e neuralgia, e para diarréia, e externamente em reumatismos, bronquites e condições inflamatórias. É largamente empregada contra gripes, resfriados e complicações inflamatórias ${ }^{3}$. Além disso, tem largo uso como incenso, anti-séptico e sedativo em problemas genital-urinários. É também usada na fabricação de nitrocelulose, materiais plásticos, produtos químicos e repelentes para insetos ${ }^{4}$. Seu preço modesto, fácil acesso e baixa toxicidade $\left(\mathrm{LD}_{50} \mathrm{em} \text { ratos: } 3000 \mathrm{mg} / \mathrm{kg}\right)^{5}$ permite sua manipulação em sala de aula sem maiores cuidados.

A estrutura molecular da cânfora foi determinada em uma época em que estudos de determinação estrutural eram inteiramente baseados em reações químicas, geralmente aquelas em que certas ligações no composto eram quebradas, dando origem a compostos sucessivamente mais simples, e cada qual era isolado, purificado e analisado. Estas sequiências de reações eventualmente levavam a substâncias de estruturas conhecidas e o caminho de volta aos compostos originais era estabelecido pelo conhecimento de mudanças estruturais que se tinha sobre uma reação particular ${ }^{6}$. A estrutura

*e-mail: kzl@rmn.uff.br

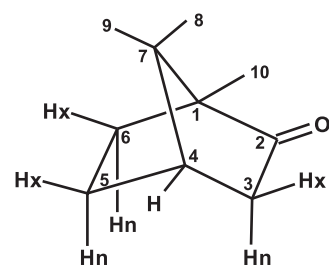

Figura 1. Cânfora $\left(\mathrm{C}_{10} \mathrm{H}_{10} \mathrm{O}\right)$

da cânfora foi particularmente difícil de ser determinada porque sistemas de anéis bicíclicos eram previamente desconhecidos e passavam por rearranjo de carbocátions, o que era uma dificuldade para os primeiros trabalhos na área daqueles que tentavam determinar suas respectivas estruturas ${ }^{7}$.

A cânfora é um bom modelo para ilustrar a aplicação da RMN na elucidação estrutural ${ }^{2}$ e é a estrutura mais citada em um dos livros texto que introduziu os químicos às modernas técnicas de $\mathrm{RMN}^{8}$. Embora seja uma molécula relativamente simples, seu rígido sistema de anel bicíclico e combinação de grupos funcionais introduzem diferentes tipos de efeitos estéricos e eletrônicos que afetam os deslocamentos químicos, constantes de acoplamento, tempos de relaxação e efeitos Overhauser nuclear (eOn $)^{8}$. A conectividade entre diferentes átomos pode ser estabelecida a partir de cálculos ou modelos moleculares utilizandose os mesmos protocolos empregados em elucidação estrutural de estruturas maiores e mais complexas, e serve para mostrar como fatores como interações internucleares, distribuição de elétrons e efeitos anisotrópicos de certos grupos funcionais afetam estes parâmetros. Além disso, a cânfora forma cristais de alta qualidade que permitem sua determinação estrutural no estado sólido por difração de raios- $\mathrm{X}$, com um nível de precisão que pode ser usado para validar cálculos teóricos e estabelecer uma base para correlações entre sua estrutura e parâmetros de RMN. Adicionalmente, um grupo carbonila é bastante conveniente para estudos de complexação com reagentes de deslocamento e relaxa- 
ção, incluindo os de natureza quiral. A cânfora tem sido usada para testar e validar muitos conceitos e experimentos que foram desenvolvidos com o passar dos anos. Também serve para ilustrar as dificuldades que foram encontradas, bem como os assinalamentos conflitantes que foram relatados na literatura desde os primórdios da RMN (e incluem trabalhos mais recentes baseados em experimentos sofisticados) ${ }^{9}$. O problema é que até em campos mais fortes, como os dos espectrômetros que operam a 14,09 Tesla (600 MHz para o hidrogênio), alguns de seus sinais estão próximos de segunda ordem, e estabelecer a conectividade apropriada requer experimentos que consomem mais tempo ${ }^{10}$. Vale notar que os efeitos de solvente nos parâmetros de RMN são significativos e podem ser responsáveis por algumas das diferenças nos assinalamentos de espectros de $\mathrm{RMN}$ de ${ }^{1} \mathrm{H}$.

A seqüência de experimentos mais simples que poderia ser utilizada para uma interpretação completa dos espectros de RMN de ${ }^{1} \mathrm{H}$ e ${ }^{13} \mathrm{C}$ da cânfora, em ordem crescente de complexidade e de tempo para realizá-los foi recentemente relatada ${ }^{11}$. As características de cada um destes experimentos, o tipo de informação que proporcionam e as diferentes maneiras de combiná-los para determinar características estereoquímicas são discutidas com o objetivo de ilustrar os conceitos que foram utilizados para este fim. Eles podem ser acompanhados por materiais selecionados em livros texto tais como as refs. 8 e 12 .

\section{PARTE EXPERIMENTAL}

A cânfora utilizada nos experimentos de RMN foi obtida da Aldrich Chemical Company Inc. As quantidades utilizadas foram: $2 \mathrm{mg}$ nos experimentos de RMN de ${ }^{1} \mathrm{H},{ }^{1} \mathrm{H},{ }^{1} \mathrm{H}-\mathrm{COSY}$ ("Correlation Spectroscopy") e ${ }^{1} \mathrm{H},{ }^{1} \mathrm{H}-\mathrm{NOEdiff}$ (NOE "difference spectroscopy") e $200 \mu \mathrm{g}$ nos experimentos de APT ("Attached Proton Test") e HETCOR ("Heteronuclear Correlation Spectroscopy"). Todos os espectros, exceto aqueles usados para efeitos de solvente, foram adquiridos em $600 \mathrm{~mL}$ de $\mathrm{CDCl}_{3}$ à $25^{\circ} \mathrm{C}$ em tubos $5 \mathrm{~mm}$ o.d. a 7,05 T $(299,96$ e $75 \mathrm{MHz}$ para ${ }^{1} \mathrm{H}$ e ${ }^{13} \mathrm{C}$, respectivamente), em um espectrômetro Varian Unity-plus $300 \mathrm{MHz}$. Os experimentos de eOn foram feitos em amostras degasadas e fechadas. Os deslocamentos químicos $\delta$ ppm são dados com relação ao TMS (em $\mathrm{CDCl}_{3}$ ) como padrão interno. Os espectros em 2D foram adquiridos com as condições padronizadas do software da Varian. O experimento de HMBC foi feito nas mesmas condições em um espectrômetro de Avance DRX 500 (Bruker) a 11.74 T.

Cálculos de mecânica molecular foram realizados com o método MM2 ${ }^{13}$ disponível no programa CHEM3D. O cálculo $a b$ initio da cânfora foi feito em estações de trabalho "SGI Octane" e "SGI Origin 2000" utilizando-se os mesmos protocolos da ref. 14; a otimização de geometria foi feita com o método Hartree Fock utilizando-se a base $6-31 \mathrm{G}^{*}$ e constantes de blindagem foram calculadas com o método GIAO no mesmo nível de teoria. O grupo carbonila da cânfora foi colocado no centro de um gradeamento de pontos, numa extensão de $-10 \mathrm{a}+10 \Delta$ nas três dimensões (espaçamento de $0,5 \Delta$ ), resultando em um cubo de 68921 pontos de grade. As coordenadas e os valores de blindagem ao redor da carbonila foram transformadas em arquivos de contorno e o efeito anisotrópico da carbonila foi visualizado através de superfícies de mesmo deslocamento químico. Deste modo, é possível mapear a extensão espacial, o sinal e alcance do correspondente efeito anisotrópico da carbonila na cânfora em cada posição estereoquímica.

\section{Interpretação do espectro a nível elementar}

A partir da integração do espectro de $\mathrm{RMN}$ de ${ }^{1} \mathrm{H}$ da cânfora em $\mathrm{CDCl}_{3}$ verifica-se que existem absorções correspondentes a um hidrogênio em 2,$35 ; 2,09 ; 1,95 ; 1,84 ; 1,68 ; 1,40$ e 1,34 ppm e correspondentes a três hidrogênios em 0,96; 0,91 e 0,84 ppm (note que a determinação precisa dos deslocamentos químicos requer um tratamento mais complexo) $)^{12}$. Estudantes com conhecimentos básicos de interpretação de espectros devem identificar prontamente os pares de hidrogênios geminais responsáveis pela grande constante de acoplamento spin-spin bem como os três simpletos entre 0 e 1 ppm que correspondem aos hidrogênios dos três grupos metila. Diferenças na blindagem podem ser associadas à proximidade ao átomo de oxigênio, porém um conhecimento de efeitos anisotrópicos do grupo carbonila seria necessário para se interpretar o espectro com base apenas nos deslocamentos químicos (ver a seção Assinalamentos com base nos deslocamentos químicos) e, assim, recorre-se a uma combinação de diferentes técnicas para se obter o assinalamento correto.

\section{Assinalamentos com base em padrões de acoplamento spin-spin}

Três tipos de padrão de acoplamento podem ser aparentemente identificados: dupletos com uma grande constante de acoplamento e acoplamentos adicionais em 2,35; 1,68; 1,40 e 1,34 ppm, um tripleto em 2,09 ppm e um dupleto em 1,84 ppm. O multipleto em 1,95 ppm não é facilmente identificado à primeira vista, enquanto as três metilas não apresentam nenhum tipo de informação de acoplamento que poderia ser utilizada em seus assinalamentos (simpletos).

A interpretação de espectros é baseada na relação entre constantes de acoplamento e estrutura química ${ }^{12}$, e deve ser comple-mentada por modelos moleculares ou "softwares" utilizados para modelagem. A análise dos padrões de acoplamento das absorções em 2,35; 2,09 e 1,84 ppm permite a associação das mesmas a $\mathrm{H}_{3 \mathrm{x}}, \mathrm{H}_{3 \mathrm{n}} \mathrm{e}_{4}{ }^{12}$. Com base na relação de Karplus entre a constante de acoplamento vicinal H,H e o ângulo diedro $\alpha^{15}$, o aparente tripleto em 2,09 ppm deve corresponder $\mathrm{a}_{4}$ uma vez que este está acoplado a dois hidrogênios similares $\left(\mathrm{H}_{3 \mathrm{x}}\right.$ e $\left.\mathrm{H}_{5 \mathrm{x}}\right)$, que formam diedros similares com a ligação $\mathrm{C}_{4}-\mathrm{H}_{4}$. Este não acoplaria com $\mathrm{H}_{3 \mathrm{n}}$ e $\mathrm{H}_{5 \mathrm{n}}$ uma vez que os ângulos diedros com suas respectivas ligações C-H são $80^{\circ}$ e $77^{\circ}$, respectivamente (estes valores podem ser obtidos por modelos moleculares ou cálculos de mecânica molecular, por exemplo) ${ }^{13}$ e a constante de acoplamento deve ser em torno de $0 \mathrm{~Hz}$. Isto significa que a absorção em 1,84 ppm $(1 \mathrm{H}, \mathrm{d})$ corresponde a $\mathrm{H}_{3 \mathrm{n}}$, uma vez que este acopla apenas com $\mathrm{H}_{3 \mathrm{x}}$, enquanto a absorção em 2,35 ppm $(1 \mathrm{H}$, dt) deve ser assinalada como $\mathrm{H}_{3 \mathrm{x}}$, que deve estar acoplado com $\mathrm{H}_{3 \mathrm{n}}$, como indicado pela grande constante de acoplamento. $\mathrm{O}$ padrão de duplo tripleto em 2,35 ppm com $\mathrm{J}_{\mathrm{HH}}=18,0 \mathrm{~Hz}$, equivalente ao do dupleto em 1,84 ppm, e em seguida com acoplamento de 4,0 Hz, equivalente à constante de acoplamento de $\mathrm{H}_{4}$, e adicional acoplamento com o $\mathrm{H}_{5 \mathrm{x}}$ confirma esta interpretação. Embora $\mathrm{H}_{3 \mathrm{x}} \mathrm{e}$ $\mathrm{H}_{3 \mathrm{n}}$ estejam ligados ao mesmo carbono, $\mathrm{H}_{3 \mathrm{x}}$ aparece em campo mais baixo que $\mathrm{H}_{3 \mathrm{n}}$ devido ao efeito anisotrópico da carbonila (ver item Assinalamentos com base nos deslocamentos químicos).

Os padrões de acoplamento dos hidrogênios remanescentes são consideravelmente mais difíceis de interpretar. Níveis de energia para sistemas de quatro spins geralmente não são facilmente calculados e sua análise requer em geral uma combinação de técnicas de desacoplamento seletivo e tratamento teórico ${ }^{16}$. Os padrões de acoplamento entre os hidrogênios dos carbonos 5 e 6 são mais complicados, devido à superposição das absorções em 1,40 e 1,34 ppm e adicional acoplamento $\mathrm{W}$ dos prótons em exo com aqueles em $\mathrm{C}_{1} / \mathrm{C}_{4}$. Alguns "softwares" podem ser utilizados para simular espectros e facilitar sua interpretação com base em deslocamentos químicos e constantes de acoplamento. Este tratamento foi feito para a cânfora na ref. 17 .

Com exceção dos hidrogênios das metilas, o espectro de RMN de ${ }^{1} \mathrm{H}$ poderia ser completamente assinalado utilizando-se o experimento de ${ }^{1} \mathrm{H},{ }^{1} \mathrm{H}$ COSY ${ }^{11}$. Uma vez que há pouca informação disponível sobre o acoplamento das três absorções abaixo de 1,0 ppm, 
seu assinalamento deve ser baseado em respectivas correlações de deslocamento químico ou eOns. A primeira possibilidade é discutida no item Deslocamentos químicos de ${ }^{13} \mathrm{C}$, enquanto a última é exemplificada no próximo item.

\section{Geometria molecular e experimentos de eOn}

Medidas de eOn são extremamente úteis na elucidação estrutural $^{12}$. No caso de moléculas pequenas, quando núcleos estão espacialmente próximos, sua irradiação leva a um aumento em suas respectivas intensidades. Uma vez que o eOn depende de taxas de relaxação e varia com o inverso da sexta potência da distância entre os respectivos núcleos, pode ser utilizado para determinar a distância entre os mesmos. EOns podem ser positivos ou negativos dependendo do caminho de relaxação dos núcleos envolvidos. Uma série de experimentos de eOn foi utilizada para estabelecer as conectividades através do espaço necessárias para assinalar o espectro de ${ }^{1} \mathrm{H}$ da cânfora ${ }^{8}$.

As distâncias internucleares entre os hidrogênios podem ser obtidas por cálculos de mecânica molecular (Tabela 1) ou estimadas por modelos moleculares. Destes dados, hidrogênios que estão espacialmente próximos e deveriam sofrer aumento da intensidade do sinal pelo efeito Overhauser podem ser identificados por experimentos como NOEdiff ou NOESY ("two-dimensional NOE spectroscopy") 12.

Tabela 1. Distâncias internucleares entre os hidrogênios da Figura 1 calculados pelo método MM2 ${ }^{13}$

\begin{tabular}{lccccccc}
\hline & $\mathrm{H}_{3 \mathrm{x}}$ & $\mathrm{H}_{3 \mathrm{n}}$ & $\mathrm{H}_{4}$ & $\mathrm{H}_{5 \mathrm{x}}$ & $\mathrm{H}_{5 \mathrm{n}}$ & $\mathrm{H}_{6 \mathrm{x}}$ & $\mathrm{H}_{6 \mathrm{n}}$ \\
\hline $\mathrm{H}_{3 \mathrm{n}}$ & 1,81 & - & - & - & - & - & - \\
$\mathrm{H}_{4}$ & 2,53 & 2,75 & - & - & - & - & - \\
$\mathrm{H}_{5 \mathrm{x}}$ & 4,35 & 3,66 & 2,51 & - & - & - & - \\
$\mathrm{H}_{5 \mathrm{n}}$ & 3,67 & 2,34 & 2,73 & 1,79 & - & - & - \\
$\mathrm{H}_{6 \mathrm{x}}$ & 4,95 & 4,34 & 4,07 & 2,36 & 2,96 & - & - \\
$\mathrm{H}_{6 \mathrm{n}}$ & 4,31 & 3,28 & 4,20 & 2,99 & 2,39 & 1,79 & - \\
$\mathrm{H}_{8}^{*}$ & 2,26 & 3,74 & 2,80 & 4,58 & 4,93 & 4,66 & 4,84 \\
$\mathrm{H}_{9}^{*}$ & 4,56 & 4,94 & 2,80 & 2,24 & 3,75 & 2,29 & 3,77 \\
$\mathrm{H}_{10} *$ & 4,39 & 4,87 & 5,06 & 4,44 & 4,88 & 2,62 & 2,83 \\
\hline
\end{tabular}

* Distância com relação ao hidrogênio da metila mais próximo para o confôrmero de menor energia

Experimentos de NOEdiff podem ser utilizados para confirmar assinalamentos prévios, bem como para distinguir entre os hidrogênios das metilas da cânfora. Neste tipo de experimento, os espectros são feitos com e sem irradiação do núcleo de interesse. Sinais negativos aparecem invertidos.

Ao se irradiar $\mathrm{H}_{3 \mathrm{x}}$ em 2,35 ppm, ocorre aumento da intensidade do sinal referente aos hidrogênios da metila em $0,84 \mathrm{ppm}$ pelo efeito Overhauser. Devido à proximidade espacial entre $\mathrm{H}_{3 \mathrm{x}}$ e os hidrogênios da metila da posição 8 , o simpleto em questão corresponde a $\mathrm{H}_{8}$. Ao se irradiar a absorção da metila em 0,96 ppm, um eOn é observado para os hidrogênios em 2,09 (já assinalado como $\mathrm{H}_{4}$ ), 1,95 e 1,68 ppm. Se a irradiação destes hidrogênios aumenta o sinal de $\mathrm{H}_{4}$, eles provavelmente são correspondentes aos hidrogênios da metila na posição 9 , que está mais próxima de $\mathrm{H}_{4}$ que os hidrogênios da metila da posição 10 (Tabela 1). Por eliminação, a absorção em $0,91 \mathrm{ppm}(3 \mathrm{H}, \mathrm{s})$ corresponde a $\mathrm{H}_{10}$. Além disso, os multipletos em 1,95 e 1,68 ppm devem corresponder a $\mathrm{H}_{6 \mathrm{x}}$ e $\mathrm{H}_{5 \mathrm{x}}$ uma vez que estes se encontram praticamente eqüidistantes da metila 9 (Tabela 1) e o aumento da intensidade de seus sinais pelo efeito Overhauser é similar. Ao se irradiar um destes sinais (multipleto $(1 \mathrm{H})$ em 1,95 ppm), observa-se eOn no multipleto em 1,34 ppm, $\mathrm{H}_{3 \mathrm{x}}$ (2,35 ppm), $\mathrm{H}_{4}(2,09$ ppm) e nos hidrogênios da metila em 0,96 ppm. Logo, o multipleto irradiado corresponde a $\mathrm{H}_{5 \mathrm{x}}$, pois este está perto de $\mathrm{H}_{3 \mathrm{x}}$ e $\mathrm{H}_{4}$ e o sinal em 1,34 ppm $(1 \mathrm{H}, \mathrm{m})$ é o seu hidrogênio geminal $\left(\mathrm{H}_{5 \mathrm{n}}\right)$, que apresen- ta o eOn mais intenso. Esta irradiação também confirma que a absorção em 0,96 ppm corresponde aos hidrogênios da metila 9. Como a absorção em 1,95 ppm é referente à $\mathrm{H}_{5 \mathrm{x}}$, aquela em 1,68 ppm $(1 \mathrm{H}, \mathrm{m})$ corresponde a $\mathrm{H}_{6 \mathrm{x}}$. Por eliminação a absorção em 1,40 ppm $(1 \mathrm{H}, \mathrm{m})$ é referente à $\mathrm{H}_{6 \mathrm{n}}$.

\section{Assinalamentos com base nos deslocamentos químicos}

Em moléculas simples, os deslocamentos químicos podem ser suficientes para a identificação de um composto e para a interpretação de seu espectro. Para moléculas mais complexas, especialmente aquelas com características estereoquímicas que contribuem para a sua atividade biológica ou sua especificidade para certas aplicações, a presença de certos átomos ou grupos funcionais servirá principalmente para distinguir entre certos núcleos. Por exemplo, a influência do grupo carbonila nos deslocamentos químicos dos hidrogênios vizinhos tem sido assunto de consideráveis debates e alguma controvérsia desde que os químicos começaram a aplicar a RMN na identificação de seus compostos. Como uma publicação recente no assunto mostrou ${ }^{16}$, a descrição padrão do efeito anisotrópico da carbonila deve ser uma das mais bem conhecidas ilustrações nos livros texto de RMN.

$\mathrm{O}$ efeito anisotrópico da carbonila deve ser o principal responsável pelas diferenças de deslocamento químico de hidrogênio da cânfora. Nestes casos, porém, a representação tradicional de um cone que indica as regiões em que um núcleo é blindado ou desblindado é difícil de ser aplicada. Aqui cálculos de deslocamento químico com base na teoria podem ser de considerável utilidade na interpretação do espectro ${ }^{11,16}$. O recente desenvolvimento de superfícies de mesmo deslocamento químico com base em cál$\operatorname{culos}^{14}$ (Figura 2) proporciona uma atrativa racionalização para as blindagens dos hidrogênios da cânfora em particular. Os dois sinais dos hidrogênios das metilas, Me-8 e Me-9 respectivamente, caem em diferentes regiões anisotrópicas como pode ser visualizado na Figura 2: Me-8 está posicionada na região de blindagem $(\delta=0,84$ ppm) enquanto Me-9 ( $\delta=0,96 \mathrm{ppm})$ não é influenciada pelo efeito anisotrópico do grupo carbonila da cânfora.

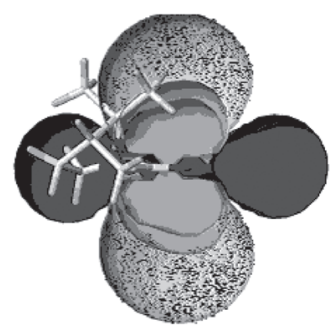

Figura 2. Efeito anisotrópico apresentado como superfícies de mesmo deslocamento químico ${ }^{14}$ do $\mathrm{H}_{2} \mathrm{CO}$ colocado na cânfora. A blindagem e desblindagem da ICSS foram calculadas pela análise de deslocamento químico independente do núcleo (NICS). Deste modo, a superfície preta corresponde a 0,1 ppm de desblindagem, a cinza claro (pontilhada) a 0,1 ppm de blindagem e a cinza escuro a 0,5 ppm de blindagem

É interessante ressaltar que os assinalamentos iniciais das metilas da cânfora foram feitos em $1958^{18}$. O assinalamento dos grupos metila foi feito com o uso de reagentes de deslocamento ${ }^{19}$ e os resultados foram consistentes com a ref. 8, onde experimentos de eOn foram realizados para este fim. Uma alternativa para a identificação dos grupos metila são os experimentos de correlação discutidos na próxima seção. Como a cânfora tem sido estudada desde os primórdios da RMN, existem muitas discrepâncias em seu assinalamento. $\mathrm{Na}$ ref. 20, por exemplo, os assinalamentos de $\mathrm{H}_{8} / \mathrm{H}_{9}$ e $\mathrm{H}_{5 \mathrm{n}} / \mathrm{H}_{6 \mathrm{n}}$ estão invertidos. Na ref. 21, não apenas os assinalamentos de $\mathrm{H}_{4} / \mathrm{H}_{5 \mathrm{x}}$, mas também de $\mathrm{H}_{5 \mathrm{n}} / \mathrm{H}_{6 \mathrm{x}}$ estão invertidos. A ref. 22 comenta que esta in- 
consistência já foi apontada na ref. 10, mas mesmo assim os erros nos assinalamentos de RMN de ${ }^{1} \mathrm{H}$ ocorreram novamente em dois trabalhos posteriores a esta referência ${ }^{9,20}$.

\section{Deslocamentos químicos de ${ }^{13} \mathrm{C}$}

Antes do uso de experimentos de pulso nos laboratórios de RMN se tornarem usuais, um esforço considerável foi investido no desenvolvimento de conceitos que poderiam ser aplicados à interpretação qualitativa dos deslocamentos químicos de ${ }^{13} \mathrm{C}$. Eles cobrem uma extensão muito maior que os deslocamentos químicos de ${ }^{1} \mathrm{H}$ e são amplamente utilizados na interpretação espectral, particularmente quando efeitos estéricos estão envolvidos ${ }^{23,24}$.

Os espectros são normalmente apresentados sem o acoplamento carbono-hidrogênio. Um experimento simples, o APT ${ }^{8}$, indica se um número ímpar ou par de hidrogênios está ligado a cada absorção de carbono. Uma vez que os deslocamentos químicos aumentam com a substituição de hidrogênios por outros átomos, um experimento de APT geralmente é suficiente para determinar o número de hidrogênios em cada carbono e confirmar que as três absorções mais blindadas $(8,8-19,3 \mathrm{ppm})$ correspondem aos grupamentos metila. Os sinais invertidos no espectro de APT são relativos a $\mathrm{CH}_{3}$ ou $\mathrm{CH}$, aquele em 42,6 ppm corresponde ao carbono terciário $\left(\mathrm{C}_{4}\right)$, o único $\mathrm{CH}$ presente na molécula, enquanto as duas absorções mais desblindadas correspondem aos carbonos quaternários. Como $\mathrm{C}_{1}$ é vizinho ao oxigênio, este deve ser mais desblindado que $\mathrm{C}_{7}$. Logo, a absorção em 57,2 ppm corresponde a $C_{1}$ e a em 46,3 ppm a $C_{7}$. Assim, uma vez que os carbonos quaternários foram assinalados, as absorções em 42,8; 29,5 e 26,7 ppm são atribuídas aos carbonos $\mathrm{CH}_{2}$. $\mathrm{O} \mathrm{CH}_{2}$ (42,8 ppm) mais desblindado deve ser $\mathrm{C}_{3}$, que está em

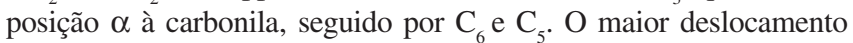
químico corresponde ao carbono da carbonila $\left(\mathrm{C}_{2}\right)$.

A mesma informação poderia ser obtida combinando-se os experimentos de ${ }^{13} \mathrm{C}$ e DEPT ("Distortionless Enhancement by Polarization Transfer") $)^{8}$, mas o tempo requerido para realizá-los seria duas vezes maior (note que a técnica ${ }^{13} \mathrm{C}$ DEPT-135 padrão não leva em conta os sinais de carbonos quaternários). Com o objetivo de se distinguir os carbonos das metilas, uma correlação heteronuclear (HETCOR $)^{12}$ ou correlação heteronuclear a múltiplas ligações (HMBC) $)^{12}$ deveria ser empregada. Do espectro de HETCOR, as absorções em 8,8; 18,7 e 19,3 ppm correlacionam-se com os hidrogênios em 0,91; 0,96 e 0,84 ppm, respectivamente, e correspondem a $\mathrm{C}_{10}, \mathrm{C}_{9}$ e $\mathrm{C}_{8}$. Um experimento de HETCOR seria suficiente para assinalar todos os núcleos de carbono, uma vez que todos os hidrogênios já foram assinalados (Tabela 2), mas o experimento de APT é muito mais fácil e rápido de ser realizado. Para aplicações simples ele pode substituir outros experimentos.

Para espectros mais complexos, um experimento de $\mathrm{HMBC}^{12}$ pode ser muito útil. Neste caso os acoplamentos entre os núcleos

Tabela 2. Dados de $\mathrm{RMN}$ de ${ }^{1} \mathrm{H}(300 \mathrm{MHz})$ e ${ }^{13} \mathrm{C}(75 \mathrm{MHz}) \mathrm{da}$ cânfora, em ppm, relativos ao TMS (solvente: $\mathrm{CDCl}_{3}$ )

\begin{tabular}{lclc}
\hline & $\delta{ }^{1} \mathrm{H}$ (mult., J) & & $\delta{ }^{13} \mathrm{C}$ (mult.) \\
\hline $\mathrm{H}_{3 \mathrm{n}}$ & $1,84(\mathrm{~d}, 18,3 \mathrm{~Hz})$ & $\mathrm{C}_{1}$ & $57,2(\mathrm{C})$ \\
$\mathrm{H}_{3 \mathrm{x}}$ & $2,35(\mathrm{dt}, 18,3 \mathrm{~Hz}, 4,4 \mathrm{~Hz})$ & $\mathrm{C}_{2}$ & $218,8(\mathrm{C})$ \\
$\mathrm{H}_{4}$ & $2,09(\mathrm{t}, 4,4 \mathrm{~Hz})$ & $\mathrm{C}_{3}$ & $42,8\left(\mathrm{CH}_{2}\right)$ \\
$\mathrm{H}_{5 \mathrm{n}}$ & $1,34(\mathrm{~m})$ & $\mathrm{C}_{4}$ & $42,6\left(\mathrm{CH}^{2}\right)$ \\
$\mathrm{H}_{5 \mathrm{x}}$ & $1,95(\mathrm{~m})$ & $\mathrm{C}_{5}$ & $26,6\left(\mathrm{CH}_{2}\right)$ \\
$\mathrm{H}_{6 \mathrm{n}}$ & $1,41(\mathrm{~m})$ & $\mathrm{C}_{6}$ & $29,5\left(\mathrm{CH}_{2}\right)$ \\
$\mathrm{H}_{6 \mathrm{x}}$ & $1,67(\mathrm{~m})$ & $\mathrm{C}_{7}$ & $46,3(\mathrm{C})$ \\
$\mathrm{H}_{8}$ & $0,84(\mathrm{~s})$ & $\mathrm{C}_{8}$ & $19,3\left(\mathrm{CH}_{3}\right)$ \\
$\mathrm{H}_{9}$ & $0,96(\mathrm{~s})$ & $\mathrm{C}_{9}$ & $18,7\left(\mathrm{CH}_{3}\right)$ \\
$\mathrm{H}_{10}$ & $0,91(\mathrm{~s})$ & $\mathrm{C}_{10}$ & $8,8\left(\mathrm{CH}_{3}\right)$ \\
\hline
\end{tabular}

de carbono e hidrogênio separados por mais do que uma ligação são correlacionados; os picos de correlação no espectro de HMBC indicam as correspondentes conectividades.

\section{CONCLUSÕES}

Com a abordagem proposta neste trabalho os alunos podem desenvolver a capacidade de raciocínio lógico utilizando uma variedade de experimentos. O poder da espectroscopia de RMN na elucidação estrutural vem da combinação de diferentes experimentos que são importantes para a determinação da estrutura completa de uma certa molécula. Experimentos isolados têm suas limitações, e é a combinação deles que determina a eficiência da RMN como ferramenta para a elucidação estrutural. Para aqueles que lecionam RMN, a cânfora é um verdadeiro presente da natureza, tanto do ponto de vista teórico quanto prático. Isto pode ser associado com muitos aspectos interessantes, que podem ser utilizados para ilustrar aulas sobre RMN.

\section{MATERIAL SUPLEMENTAR}

Os espectros de RMN de ${ }^{1} \mathrm{H}$, NOE diff., APT, HETCOR e HMBC da cânfora bem como uma figura ilustrando os padrões de acoplamento entre os hidrogênios $\mathrm{H}_{3,}, \mathrm{H}_{3 \mathrm{n}}$ e $\mathrm{H}_{4}$ estão disponíveis em http://quimicanova.sbq.org.br, em arquivo PDF, com acesso livre.

\section{AGRADECIMENTOS}

À CAPES e ao programa PRH da ANP/FINEP.

\section{REFERÊNCIAS}

1. Pinto, G.; J. Chem. Educ. 2005, 82, 1321.

2. Alty, L. T.; J. Chem. Educ. 2005, 82, 1387.

3. http://botanical.com/botanical/mgmh/c/campho13.html, acessada em Outubro 2007.

4. Enciclopédia Barsa; Houaiss, A.; Barbosa, F. A., eds.; Encycopaedia Britannica do Brasil, RJ-SP, 1990, vol. 5.

5. Merck Index; $12^{\text {th }}$ ed.; Budavari, S., ed.; Whitehouse Station: New Jersey, 1996.

6. Macropaedia; Isoprenoids, Encyclopaedia Britannica, 1977, vol. 9.

7. Roberts, J. D.; Caserio, M.; Basic Principles of Organic Chemistry, $2^{\text {nd }}$ ed., Benjamin, Menlo Park: USA, 1977, chapter 30.

8. Sanders, J. K. M.; Hunter, B. K.; Modern RMN Spectroscopy- a guide for chemists, Oxford University Press: Oxford N.V. Toronto, 1988, p. 192-193; $252 ; 253-256$

9. Waterhouse, A. L.; Magn. Reson. Chem. 1989, 27, 37.

10. Yu, C.; Levy, G. C.; J. Am. Chem. Soc. 1984, 106, 6533.

11. Yoneda, J. D.; Seidl, P. R.; Leal, K. Z.; Pimentel, H. R. X.; Ann. Magn. Reson. 2003, 2, 52

12. Friebolin, H.; Basic One and Two-Dimensional RMN Spectroscopy, $3^{\text {rd }}$ ed., Wiley- VCH: Chichester, 1998, chapters 3, 4, 10.

13. Burkert, U.; Alinger, N. L.; Molecular Mechanics, ACS Monograph 177, American Chemical Society; Washington, DC, 1982.

14. Kleinpeter, E.; Klod, S.; J. Am. Chem. Soc. 2004, 126, 2231.

15. Gunther, H.; NMR Spectroscopy, $2^{\text {nd }}$ ed., Wiley: Chichester, 1994.

16. Abraham, R. J.; Ainger, N. J.; J. Chem. Soc., Perkin Trans. 2 1999, 27, 441.

17. Yoneda, J. D.; Seidl, P. R.; Leal; K. Z.; trabalho não publicado .

18. Kumler, W. D.; Shoolery, J. N.; Brutcher, Jr., F. V.; J. Am. Chem. Soc. 1958 , 80, 2533.

19. Hinckley, C. C.; J. Org. Chem. 1970, 35, 2834

20. Abraham, R. J.; Barlow, A. P.; Rowan, A. E.; Magn. Reson. Chem. 1989, 27, 1074.

21. Grant, D. M.; Curtis, J.; Croasmun, W. R.; Dalling, D. K.; Wehrli, F. W.; Wehrli, S.; J. Am. Chem. Soc. 1982, 104, 4492.

22. Kaiser, C. R.; Rittner, R.; Basso, E. A.; Magn. Reson. Chem. 1994, 32, 503.

23. Seidl, P. R.; Leal, K. Z .; Yoneda, J. D.; J. Phys. Org. Chem. 2002, 15, 801 .

24. Seidl, P. R.; Yoneda, J. D.; Leal, K. Z.; J. Phys. Org. Chem. 2005, 18, 162. 


\section{CÂNFORA: UM BOM MODELO PARA ILUSTRAR TÉCNICAS DE RMN}

Julliane Diniz Yoneda e Kátia Zaccur Leal*

Departamento de Físico-Química, Instituto de Química, Universidade Federal Fluminense, Outeiro de São João Batista, s/n, 24020-150 Niterói - RJ, Brasil

\section{Peter Rudolf Seidl}

Departamento de Processos Orgânicos, Escola de Química, Universidade Federal do Rio de Janeiro, 21949-900, Ilha do Fundão, Rio de Janeirp - RJ, Brasil

Rodrigo Bagueira de V. Azeredo

Departamento de Química Orgânica, Instituto de Química, Universidade Federal Fluminense, 24020-150 Niterói - RJ, Brasil Erich Kleinpeter

Universität Potsdam, Chemisches Institut, P.O. Box 6015 53, D-14415, Potsdam, Germany

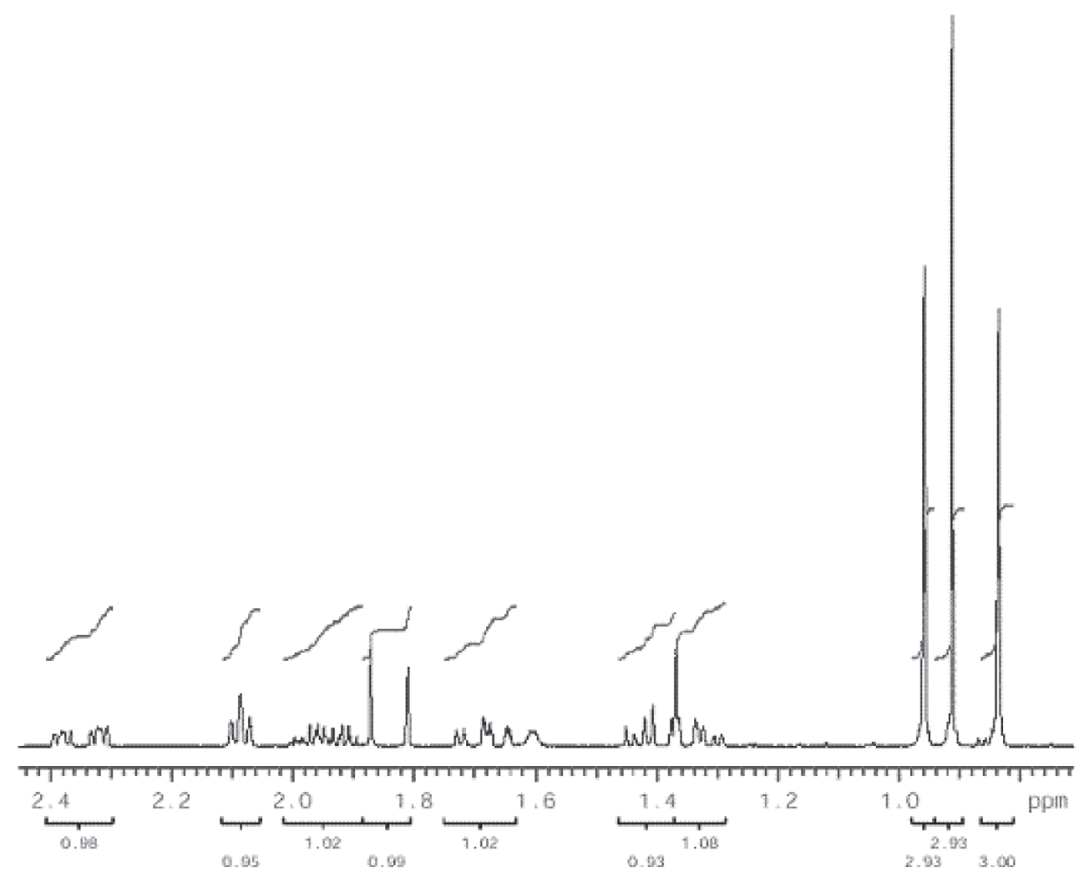

Figura 1S. Espectro de RMN de ${ }^{l} \mathrm{H}$ da cânfora a 7,05 T

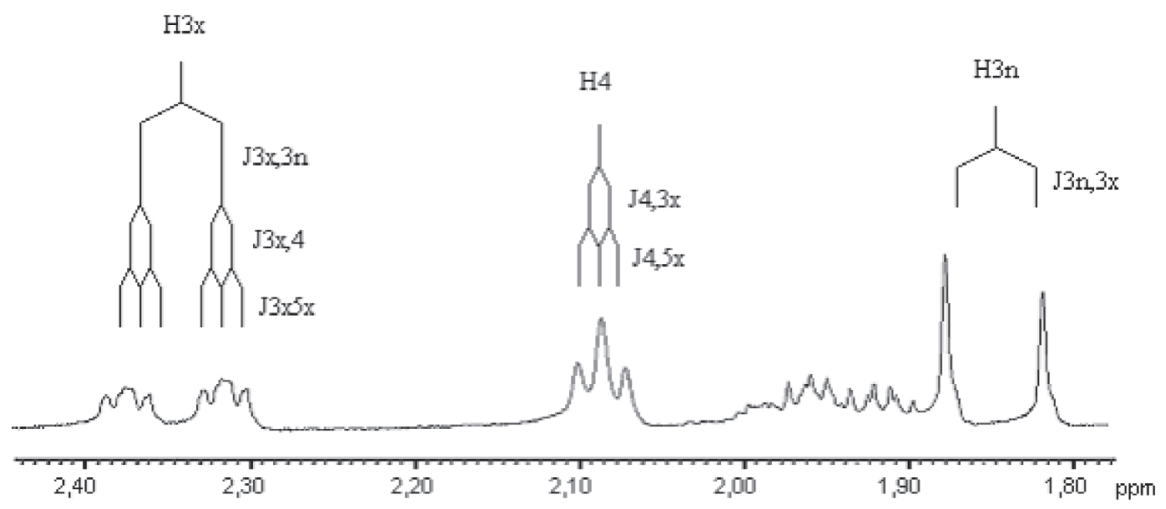

Figura 2S. Padrões de acoplamento entre os hidrogênios $H_{3 x} H_{3 n}$ e $H_{4}$ da cânfora

*e-mail: kzl@rmn.uff.br 

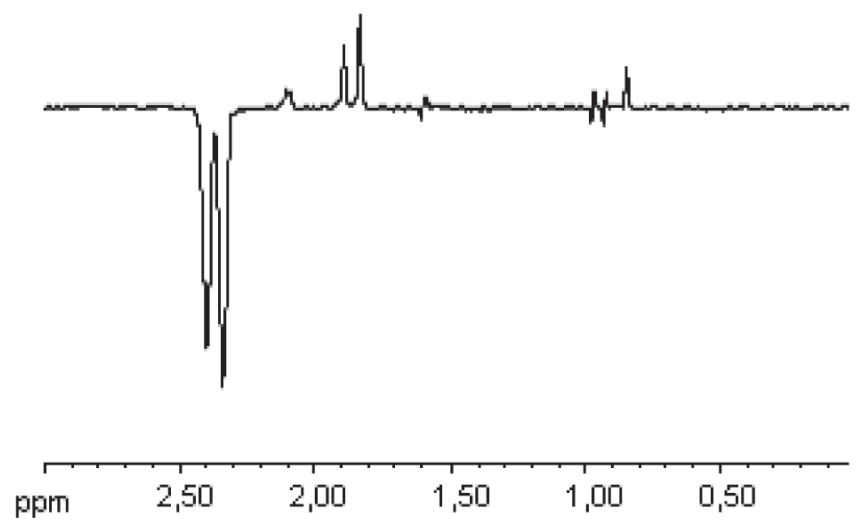

Figura 3S. Espectro de NOE diff. para irradiação em 2,35 ppm

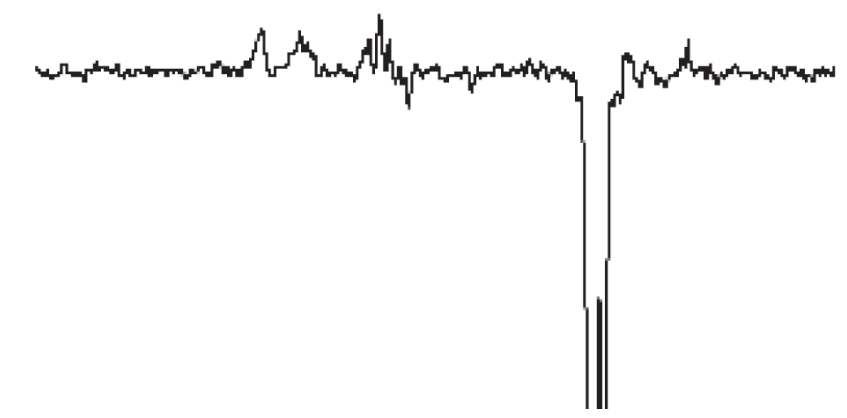

$\begin{array}{llllll}\text { pprm } & 2,50 & 2,00 & 1,50 & 1,00 & 0,50\end{array}$

Figura 4S. Espectro de NOE diff. para irradiação em 0,96 ppm

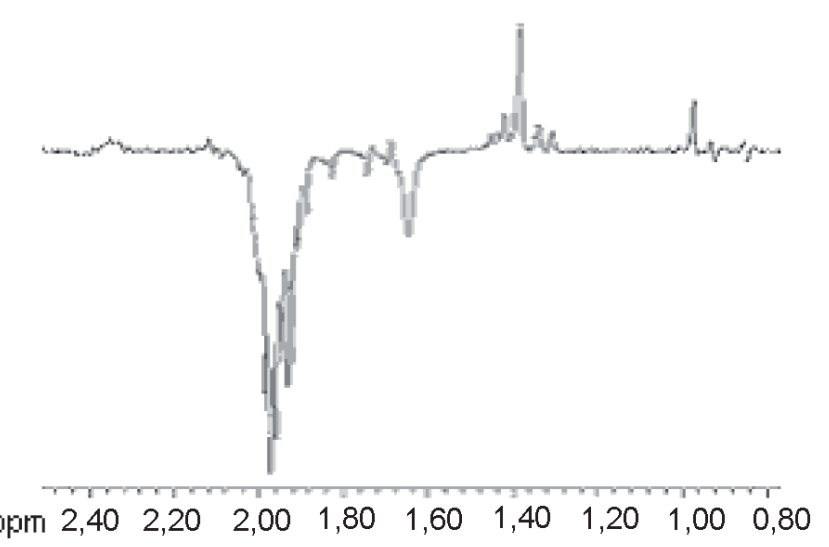

Figura 5S. Espectro de NOE diff. para irradiação em 1,95 ppm

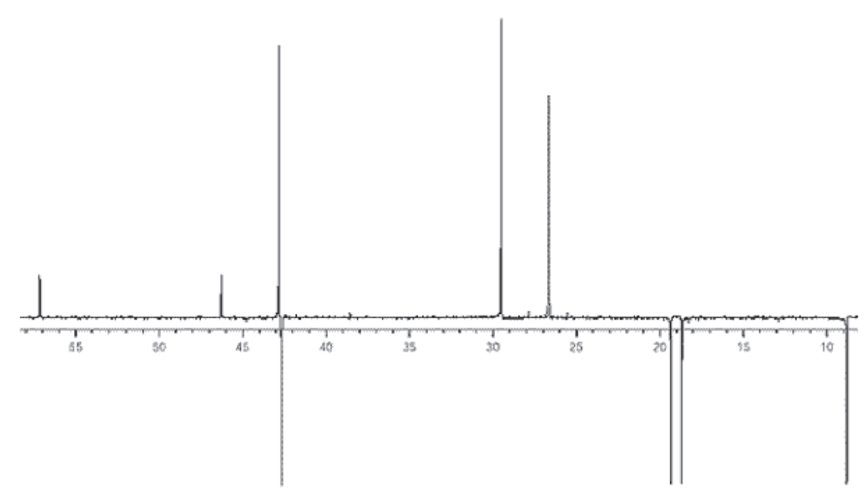

Figura 6S. Espectro de APT da cânfora a 7,05 T 


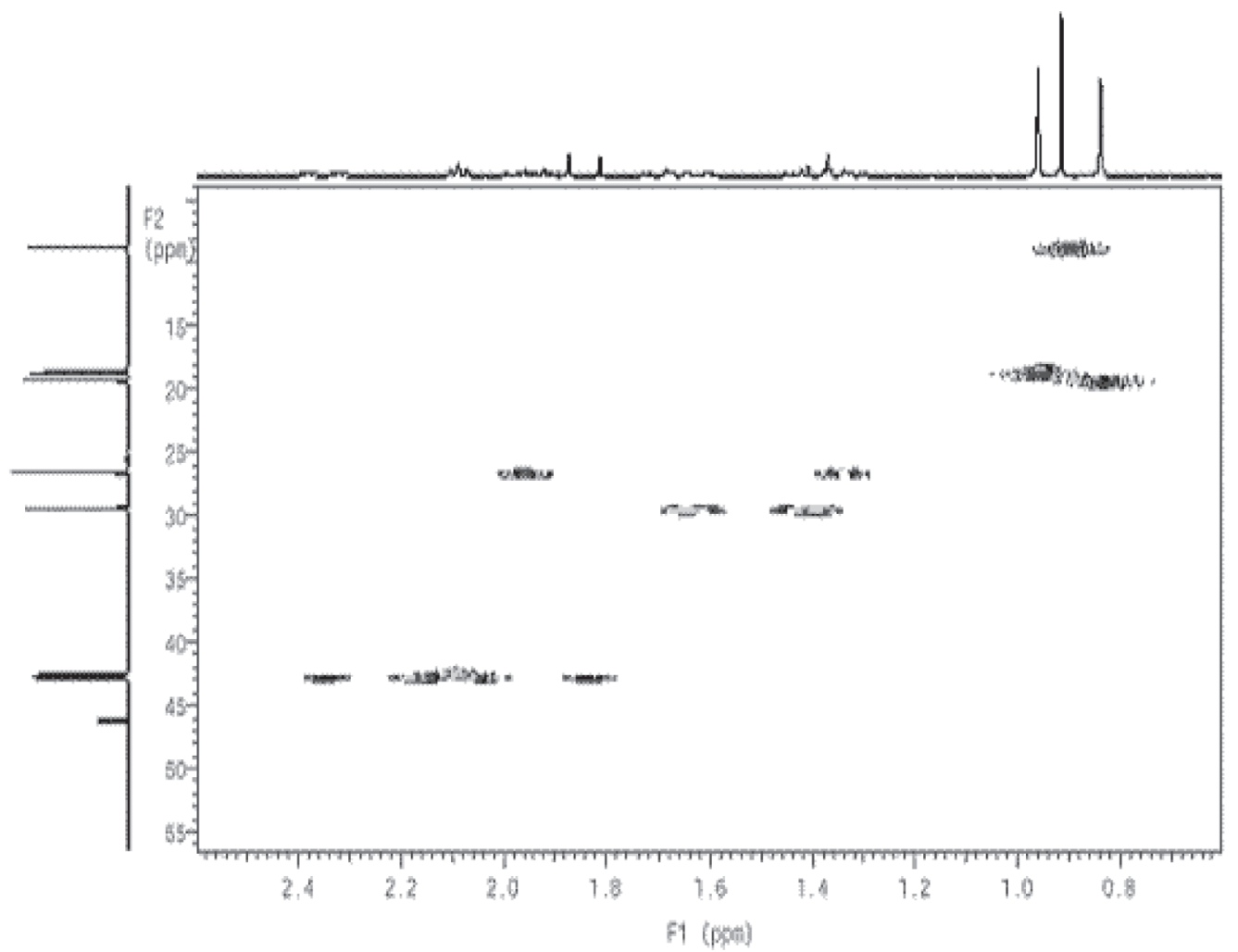

Figura 7S. Espectro de HETCOR da cânfora a 7,05 T

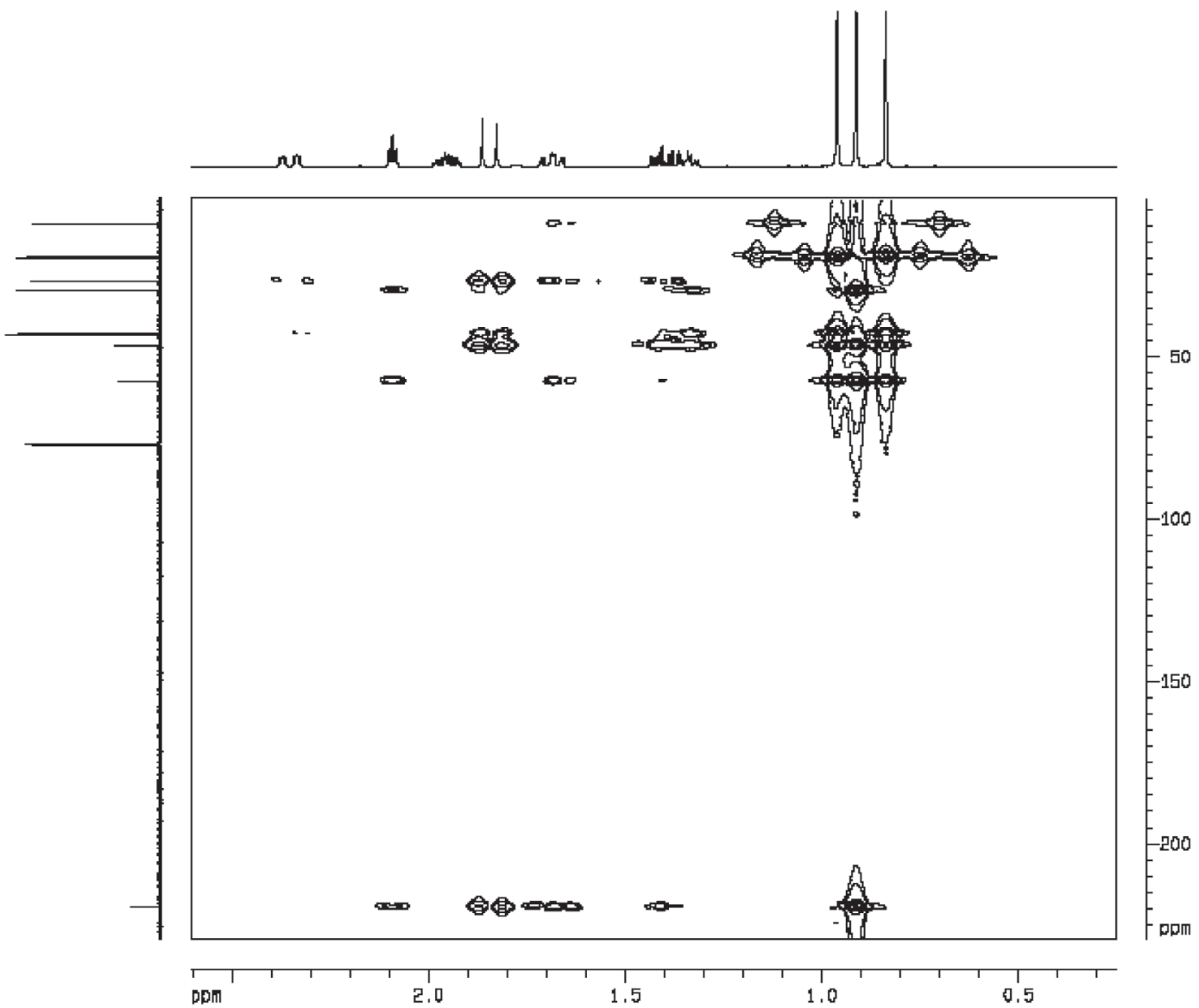

Figura 8S. Espectro de HMBC da cânfora a 11,74 T 